\title{
Temporal characteristics of noise conditions producing facilitation and interference
}

\author{
G. ROBERT GRICE and JOHN W. GWYNNE \\ University of New Mexico, Albuquerque, New Mexico
}

\begin{abstract}
In reaction time (RT) experiments, a target letter was flanked by noise letters with differing relations to the target. When noise letters that are identical to the target, or response-compatible but not identical, precede the target by $100 \mathrm{msec}$, RT is facilitated. Facilitation by identical noise is greater. Scaling analysis of the RT distributions indicated that facilitation by compatible noise is a very short-latency, transient effect. Facilitation by identical noise is more persistent. It is suggested that there are two facilitative processes-direct response priming by compatibility, and facilitation of stimulus coding by stimulus identity. Facilitation is not obtained when these noise letters are presented simultaneously with the target. Associative interference is produced by response-incompatible noise letters, both with simultaneous presentation and with noise preceding the target. Maximum interference is earlier when noise precedes the target. This confirms previous findings and a prediction of continuous growth theory.
\end{abstract}

The reaction time (RT) paradigm introduced by B. A. Eriksen and C. W. Eriksen (1974), in which the target letter is flanked by noise letters bearing differing relations to the target, has proved to be both heuristic and fruitful in subsequent research. Recently, Grice, Boroughs, and Canham (1984) investigated the temporal dynamics of associative facilitation and interference in this situation. Their approach to this problem was twofold. First, they manipulated the stimulus onset asychrony (SOA) between target and noise as had been done in previous research by C. W. Eriksen and Schultz (1979) and by Flowers and Wilcox (1982). Second, they investigated the temporal dynamics within the trial at each SOA by employing the scaling methods of variable criterion theory to the cumulative RT distribution functions. As measured by average reaction time, they found that, when the noise preceded the target by $250 \mathrm{msec}$, the associative interference effect produced by response-incompatible noise letters decreased substantially from what it was when simultaneous presentation or shorter SOAs were used. Noise letters identical to the target did not produce facilitation when they occurred simultaneously with the target, but actually produced a small distraction effect when compared with a no-noise control condition. However, identical noise letters did produce facilitation when they preceded the target by as little as $50 \mathrm{msec}$. This facilitation increased to a maximum at $100 \mathrm{msec}$ and was about the same at 250 msec.

Functions for the time course of facilitation and interference were measured in scale units as components of the functions for the growth of excitatory strength. The amounts of interference were nonmonotonic functions of

Requests for reprints should be sent to G. Robert Grice, Department of Psychology, University of New Mexico, Albuquerque, NM 87131. time following stimulus onset, with maximum interference occurring progressively earlier as the SOA by which the response-incompatible noise letter preceded the target was increased. Facilitation was primarily a shortlatency effect that influenced the early portion of the RT distribution. As the SOA by which the identical noise letter preceded the target was increased, facilitation began still earlier. The dependence of both interference and facilitation upon the SOA is consistent with predictions of the continuous growth principle used in variable criterion theory and with the continuous flow model of C. W. Eriksen and Schultz (1979).

The primary purpose of the present research was to extend this kind of analysis to the effects of noise letters that are response-compatible with the target, but not identical to it. In their investigation of SOA, C. W. Eriksen and Schultz (1979) included both kinds of compatible noise, but they were primarily concerned with the comparison of response-compatible and response-incompatible noise and did not separately analyze the effects of the two kinds of compatible noise. In a somewhat more complex experiment, Flowers and Wilcox (1982) did provide such an analysis. When noise preceded the target, they found that both identical and nonidentical compatible noise produced facilitation when compared with a neutral noise control. The effect of an identical noise letter or digit was a little greater in the range of 67-to-200-msec SOA. With simultaneous presentation, however, RTs were faster with compatible than with identical noise. RTs were also faster with nonidentical, compatible noise than in the neutral noise condition, which used asterisks. A no-noise control condition was not included. The findings led the authors to conclude that, with simultaneous presentation, facilitation was produced by compatible noise but not by identical noise. There is reason, however, to question the generality of these findings. C. W. Eriksen and B. A. 
Eriksen (1979) found the compatible noise condition to be slower than the identical noise condition with simultaneous presentation of target and noise. The present experiments provide additional evidence concerning this inconsistency. They were also expected to provide evidence concerning temporal dynamics within SOAs from scaling analysis of the cumulative RT distribution functions.

Another concern here was the identification of an adequate control condition for the evaluation of facilitation and distraction effects. In their Experiment 1, Grice, Boroughs, and Canham (1984) used a no-noise control at all SOAs. However, with increasing SOAs, RTs in this condition increased while those in the other conditions decreased. This suggested that noise letters preceding the target have a warning-signal effect, and that in the no-noise condition subjects were less well prepared. Thus, the control was appropriate only at 0 SOA. As a result, in Experiment 2, parentheses were used in the positions of the noise letters with the same SOAs. This eliminated the problem of increasing RTs with increasing SOAs. However, there was some evidence that, at 0 SOA at least, the parentheses produced a small interference or distraction effect. The solution attempted here was to use a no-noise control condition, but to turn off the fixation point at the same time that the noise letters would appear. This should provide a warning comparable to the onset of noise letters. In addition, to allow for comparison with the previous research, the experiments were also replicated with the parentheses. In addition to the no-noise and nonidentical compatible conditions, the remaining conditions included were identical noise, irrelevant noise, and response-incompatible noise. This was done to permit comparison of the interference or facilitation effects produced by compatible noise with those found in previous studies, as well as to provide replication of these conditions and their analyses.

The research consisted of two main experiments, each composed of two subexperiments. The subexperiments were based on separate groups of subjects, and each contained the five noise conditions, presented in an irregular order. The two main experiments differed only in the condition without flanking noise letters. In the first, parentheses were used in these positions; in the second, the noise positions were blank. The procedures used in the subexperiments were simultaneous presentation of target and noise and noise preceding the target by $100 \mathrm{msec}$. The SOA of $100 \mathrm{msec}$ was selected because it had produced the maximum facilitation by identical noise in the previous research.

\section{METHOD}

\section{Subjects}

The subjects were 112 undergraduate women enrolled in courses in introductory psychology. They received course credit for participation. There were 28 subjects in each of the four subexperiments.

\section{Apparatus and Procedure}

The experimental situation and procedures have been described fully by Grice, Boroughs, and Canham (1984) and by Grice, Canham, and Schafer (1982). The experiments were controlled and data recorded by a TRS-80 Model I microcomputer equipped with interface devices and a millisecond clock. Stimuli were presented on a separate video monitor, and responses were made with the left and right forefingers operating two telegraph keys. Letter stimuli were approximately $.53^{\circ}$ high and $.43^{\circ}$ wide. The fixation point, a plus sign, appeared in the center of the screen at all times except during stimulus presentation. The target letter appeared $.45^{\circ}$ directly above the fixation point. When noise letters were presented, the same letter appeared on each side of the target with an edge-toedge separation of $.5^{\circ}$.

There were four target letters, two mapped on each of the two responses. One half of the subjects responded to the letters $H$ and $\mathrm{K}$ with the left key and the letters $\mathrm{C}$ and $\mathrm{S}$ with the right key. The other half of the subjects had the reverse mapping. A .5-sec auditory warning signal preceded stimulus onset by $1.0 \mathrm{sec}$. The subjects were instructed to look at the fixation point when the warning signal sounded and to respond to the target as quickly as possible while avoiding errors. They were also instructed to ignore the flanking noise letters. Visual and auditory feedback was given for errors. There were five noise conditions in each experiment: no noise letter, noise letter identical to the target, noise letter the other member of the response set of the target (compatible), noise letter irrelevant (the letter $\mathrm{Y}$ ), and noise letter response-incompatible (a member of the competing response set). The two incompatible letters were used equally often with each target, and each target appeared equally often. There were equal numbers of each noise condition, and the order of all events was irregular.

In each experiment there were two SOA conditions with separate groups of subjects. These were target and noise simultaneous and noise preceding target by $100 \mathrm{msec}$. In Experiment 1, left and right parentheses replaced the left and right noise letters, respectively, in the condition without noise letters. In Experiment 2, these positions were blank. In all instances, the fixation point went off when the noise letters appeared, or when they would have appeared if it had been a noise trial. All stimuli went off and the fixation point reappeared when the subject responded. There were $4 \mathrm{sec}$ between trials. The experiment began with 50 practice trials followed by a brief rest. There were then 250 trials without interrruption. Data were based on the final 240 of these trials, 48 with each noise condition. Data were recorded in milliseconds.

\section{RESULTS}

\section{Mean Reaction Time}

The means of subject mean RTs are presented in Figure 1 for all experimental conditions in the two experiments. Aside from the differing relationships shown by the two control conditions without noise letters, the results are quite similar. An analysis of the other four noise conditions across experiments and SOAs indicates that there were no significant effects attributable to experiments [for experiments, $F(1,108)=1.02$, n.s.; for experiments $\times$ SOA, $F(1,108)<1$; for both noise $X$ experiments and noise $\times$ SOA $\times$ experiments, $F(3,324)$ $<1]$. In other words, the difference between these two control conditions did not significantly affect the other aspects of the experiments. Error rates were low. For all noise conditions except incompatible noise, the mean rates ranged from .007 to .025 , with a mean of .015 . For incompatible noise, they ranged from .047 to .061 , with a mean of .055 . 


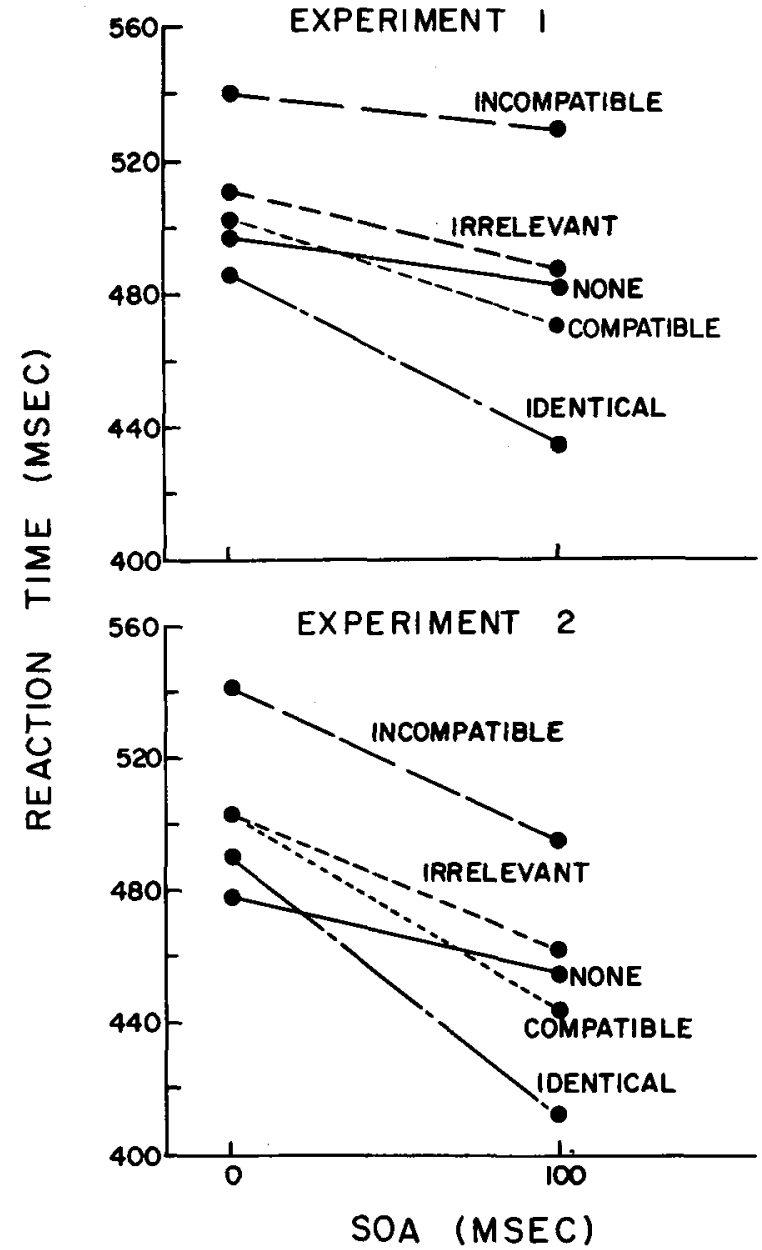

Figure 1. Means of subject mean reaction times for each experimental condition in Experiments 1 and 2.

An initial question is whether the parentheses produced a distraction effect. This was indicated by the data of Grice, Boroughs, and Canham (1984), and in the present study the parentheses condition was slower than the nonoise control. A direct test is of borderline significance $[F(1,108)=3.36, .10>p>.05]$. Other comparisons suggest the effect more strongly. For example, at 0 SOA, the parentheses condition was slower, but not significantly so, than the identical-noise condition, and the no-noise condition was faster than identical noise. This interaction is significant $[F(1,54)=10.16, p<.005]$. The no-noise condition of Experiment 2 did not show an increase in RT with $100-\mathrm{msec}$ SOA as did that of Grice, Boroughs, and Canham (1984). This suggests that, with the present procedure, this condition provided an adequate, or at least better, control for the evaluation of distraction and facilitation.

The previous finding of an interference or distraction effect by irrelevant noise letters was confirmed by these experiments. In Experiment 1, irrelevant noise was significantly slower than in the parentheses control condition $[F(1,54)=6.10, p<.025]$. Although the differ- ence is smaller at 100 SOA, the interaction is not significant. In Experiment 2, irrelevant noise was significantly slower than in the no-noise condition $[F(1,54)=$ $21.42, \mathrm{p}<.001]$. In this instance, the interaction of noise $\times$ SOA is significant $[F(1,54)=6.66, p<.025]$. This confirms the previous finding that the distraction effect tends to decrease with increasing SOA.

The well-established associative-interference effect produced by response-incompatible noise was also confirmed in these experiments, as evaluated by a comparison of the incompatible and irrelevant noise conditions. Since both conditions had noise letters different from the target, both should involve the distraction effect. The difference between them is the net interference due to response incompatibility. This difference was evaluated for both experiments together. The difference between irrelevant and incompatible noise is significant $[\mathrm{F}(1,108)$ $=125.29, \mathrm{p} .<.001]$. As indicated by the noise $\times$ experiments interaction, the effect does not differ between the two experiments $[F(1,108)<1]$. It also does not differ significantly between the two SOAs, as indicated by the noise $\times$ SOA interaction $[F(1,108)<1]$. In Experiment 1 , the effect was larger at 100 SOA than with simultaneous presentation, but in Experiment 2, it was slightly smaller with 100 SOA. However, as indicated by the three-way interaction, noise $\times$ SOA $\times$ experiments, this difference is not significant $[\mathrm{F}(1,108)=2.30$, n.s. $]$. In the two conditions combined, RT is faster at $100 \mathrm{SOA}$ than at 0 SOA $[F(1,108)=5.52 \mathrm{p}<.025]$. This probably indicates that noise letters preceding the target have a warning-signal effect as well as interference effects.

Because of the distraction produced by the parentheses, the effects of identical noise are best evaluated with respect to the no-noise condition of Experiment 2. At 0 SOA, nonoise was the faster of the two conditions, and the difference is significant $[t(27)=2.484, p<.02]$. This confirms the somewhat surprising finding of Grice, Boroughs, and Canham (1984) that noise letters identical to the target are distracting when presented simultaneously with the target. When identical noise letters preceded the target by $100 \mathrm{msec}$, substantial and significant facilitation was obtained $[t(27=9.786, p<.001]$. As would be expected, the noise $\times$ SOA interaction is significant $[F(1,54)$ $=69.08, \mathrm{p}<.001]$. Facilitation was also obtained at 100 SOA, as compared with the parentheses condition $[t(27)=6.964, p<.001]$. With 0 SOA, identical noise was faster than the parentheses control, but the difference is not significant $[\mathrm{t}(27)=2.049, \mathrm{n} . \mathrm{s}$.]. In the corresponding condition of Grice, Boroughs, and Canham (1984), the parentheses condition was $2 \mathrm{msec}$ faster than identical noise. These results fully confirm the finding of Grice, Boroughs, and Canham (1984) that identical noise produces facilitation when it precedes the target but interference when presented simultaneously.

There are two meaningful comparisons for evaluating the effects of compatible noise consisting of the other member of the target response set. Interference or facilitation may be inferred from comparisons with the no-noise 
control of Experiment 2. However, this comparison may not be adequately sensitive for detecting facilitation. Since the compatible letter is different from the target, a distraction effect may also be expected. Facilitation would be implied if compatible noise were faster than irrelevant noise, which also differs from the target but does not have the response-compatible relationship. In comparison with the no-noise condition of Experiment 2, compatible noise is slower than no noise with simultaneous presentation and faster with $100-\mathrm{msec}$ SOA. This noise $\times$ SOA interaction is significant $[\mathrm{F}(1,54)=18.51, \mathrm{p}<.001]$. The interference or distraction effect at 0 SOA is significant $[\mathrm{t}(27)=3.975 \mathrm{p}<.001]$. Also, the facilitation effect at 100 SOA approaches significance $[t(27)=1.963$ $\mathrm{p}<.05$, one-tailed test]. This comparison with the nonoise control clearly indicates that a compatible noise letter has different effects, depending on whether it is presented simultaneously with the target or precedes it by $100 \mathrm{msec}$. The comparison with irrelevant noise was made for both experiments jointly. With 0 SOA, mean RT was the same for compatible and irrelevant noise in Experiment 2, and they did not differ significantly in Experiment $1[\mathrm{t}(27)=$ 1.629 , n.s.]. With 100 SOA, compatible noise was significantly faster than irrelevant noise $[F(1,54)=15.03$, $\mathrm{p}<.001]$. The resulting SOA $\times$ noise interaction is significant $[F(1,108)=4.76, \mathrm{p} .<.05]$. None of the effects involving experiments are significant. The data indicate that, with simultaneous presentation, compatible noise does not produce facilitation, but has an interference effect about equal to that of irrelevant noise. When compatible noise preceded the target by $100 \mathrm{msec}$, facilitation was obtained, but was less than that produced by identical noise.

\section{Scaling Analysis}

According to the variable criterion theory, excitatory strength leading to response evocation grows as a monotonic function of time following stimulus onset, and when this strength reaches a criterion value the response occurs. The criterion is normally distributed on the strength dimension, and the form of the growth function, together with the criterion distribution, generates the cumulative RT distribution function. For experimentally obtained distributions, it is possible to obtain estimates of the forms of the growth functions, in a Thurstonean metric, by the use of scaling procedures (see, e.g., Grice et al, 1982). When the functions for two conditions plot linearly against each other in a response evocation characteristic (REC), it implies that the two functions are of the same shape and differ only in the criterion parameters. These, the mean and standard deviation of the criterion distribution, are estimated by the parameters of the linear function. When the functions for two conditions are not linearly related, and one of the conditions involves a process logically not present in the other, the time course of this process may be estimated from the difference between the functions. One limitation is that if a process is simply additive or subtractive, as distraction effects have been found to be , the method does not differentiate it from a differ- ence in criterion means. These procedures were used by Grice, Boroughs, and Canham (1984), and were applied to the present data.

The analysis was applied to group cumulative distribution functions, averaged at 20 -msec intervals for each experimental condition. As in the preceding work, the distributions were corrected for errors by the race model of Grice, Spiker, and Nullmeyer (1979). Such corrected distributions are of unit area, and are invariant in form with respect to error rate. In this analysis, it was found that the functions for all noise conditions, except those involving associative facilitation or associative interference, were of the same shape, and differed only by linear transformations. To obtain a quantitative function describing them, the functions for no-noise at 0 and 100 SOA in Experiment 1 were converted to a common scale by an REC relating them, and then averaged. The resulting smooth, negatively accelerated function was then fitted with an exponential growth function of the form used by Grice, Boroughs, and Canham (1984). Functions for all of the remaining conditions of this class were then obtained by the parameters of linear RECs relating them to calculated values of the fitted function.

Graphs of all fitted functions for Experiment 2 are presented in Figure 2. For 0 SOA, the growth functions
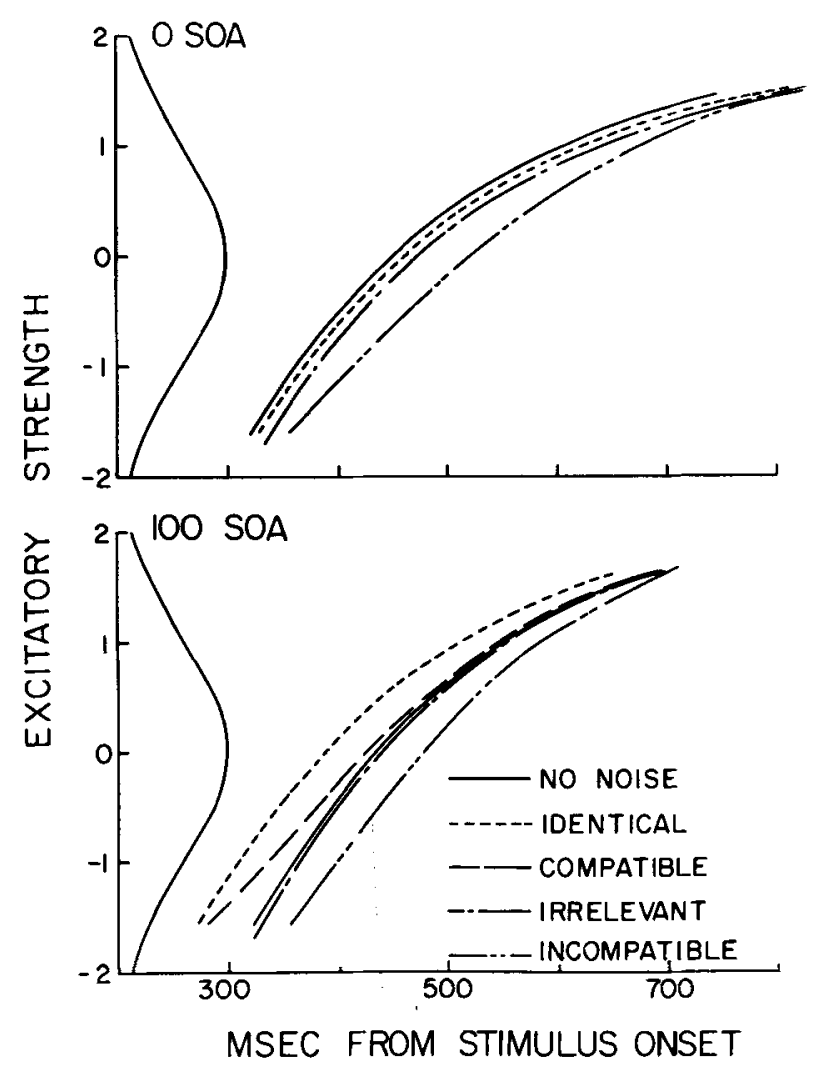

Figure 2. Calculated functions describing the growth of excitatory strength for the experimental conditions of Experiment 2. The normal density functions on the strength axis illustrate the relation of the criterion distribution to these functions. The standard deviation of the criterion distribution is the unit of the strength dimension. 
for identical, compatible, irrelevant, and no noise are of the same shape and differ by additive constants. The nonoise control has the greatest strength throughout the range, and the separation from identical noise represents the small distraction effect of identical noise. The further separation of irrelevant noise represents the distraction effect of that condition. The function for compatible noise is almost identical to that for irrelevant noise and cannot be represented separately. This represents the finding that compatible and irrelevant noise had the same distraction effect, indicated by approximately the same subtractive constant. For 100 SOA, only the functions for no noise and irrelevant noise are of this same shape. Since the distraction produced by irrelevant noise was very slight with $100 \mathrm{SOA}$, the two functions are very nearly the same. Calculated functions for the remaining conditions are described below. The normal density functions on the strength axis represent the criterion distribution.

Since response-incompatible noise letters presumably both have the distraction effect and produce associative interference, the time course of associative interference is inferred from the difference between the functions for incompatible and irrelevant noise. The shapes of the incompatible-noise functions were about the same in the two experiments, so the function for Experiment 1 was transformed to the scale of Experiment 2 by the parameters of the linear REC relating them. Means of the two functions were then obtained at 20-msec intervals. The functions for associative interference are the differences between these means and the calculated values of the function for irrelevant noise. The results are smooth nonmonotonic functions for both SOAs. As in the analysis of Grice, Boroughs, and Canham (1984), they were described by functions based on the derivative of the Gompertz growth function. Graphs of these functions are presented in the lower portion of Figure 3. The maximum interference occurred earlier with 100 SOA than with simultaneous presentation. This confirms the finding of Grice, Boroughs, and Canham (1984) and the prediction of continuous growth theory that interference should begin progressively sooner the more the incompatible noise precedes the target.

Functions for the time course of associative facilitation with 100 -msec SOA were estimated in a manner similar to the procedure used for interference, rather than by the less analytic method used by Grice, Boroughs, and Canham (1984). The scale values for identical and compatible noise for Experiment 1 were converted to the scale of Experiment 2 by the linear RECs, relating them to the corresponding functions of that experiment. The functions were averaged, and differences were obtained between the average functions and the no-noise function at $20-\mathrm{msec}$ intervals. Both kinds of facilitation decreased monotonically throughout the responding interval, and could be accurately described by simple exponential decay functions. These fitted functions are presented in the upper portion of Figure 3. Facilitation by compatible noise is largely limited to short latencies in the RT distribution, and it decreases rapidly with the passage of time. Identical noise,
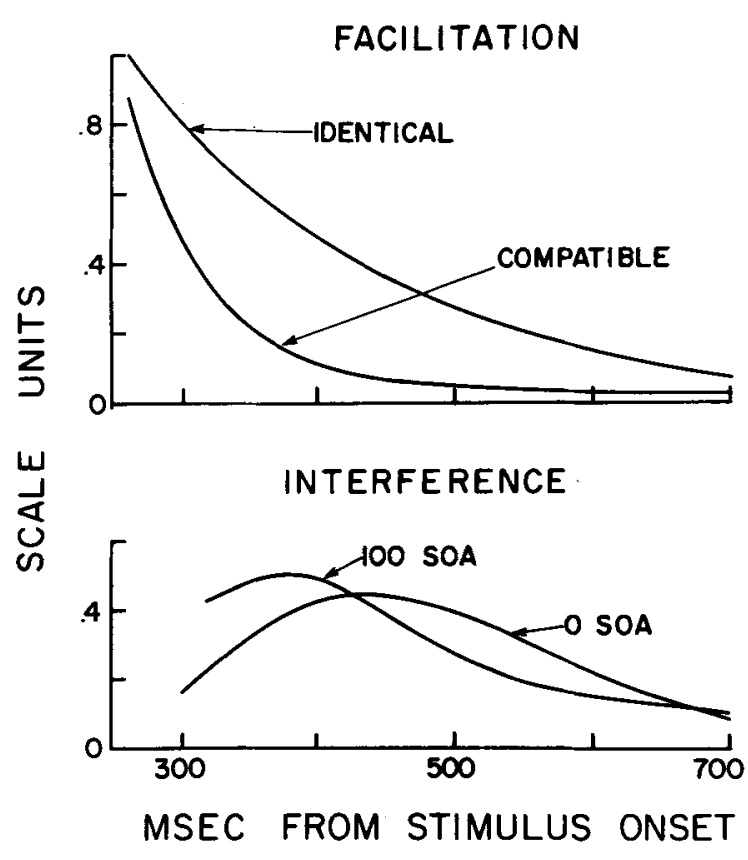

Figure 3. The upper figure for facilitation applies to the 100-msec conditions only. The curves are the calculated functions representing the time course of facilitation by identical and compatible noise letters. The curves of the lower figure are the calculated functions for the time course of associative interference for 0 and 100 SOA. All functions are on the scale of Experiment 2.

on the other hand, continues to produce substantial facilitation for a much greater time.

The calculated growth functions for incompatible, identical, and compatible noise are included in Figure 2. The functions for incompatible noise consist of those for irrelevant noise minus the interference functions. Functions for identical and compatible noise consist of that for no noise plus those for facilitation. The calculated functions provide excellent fits to the obtained scale values. The differing persistence of identical and compatible facilitation is clearly evident. The earlier interference at 100 SOA is also observable.

The adequacy of the set of calculated functions in describing the data was evaluated by fitting the 20 cumulative distribution functions on which the scaling analysis was based. The normal functions in Figure 2 illustrate the relation of the criterion distribution to the growth functions. The calculated proportion of responses at any time is the area of the normal function below the ordinate of a growth function at that time. Fitted in this way, the proportions of variance of the obtained cumulative proportions accounted for by the calculated values varied from .998 to .9997 for the 20 distributions. The mean proportion of variance accounted for was $\mathbf{9 9 9 0}$. This interpretative model provides an excellent description of the data.'

\section{DISCUSSION}

When noise letters that were response-compatible with the target, but not identical to it, preceded the target by 
$100 \mathrm{msec}$, facilitation of RT was produced. This confirms the results of Flowers and Wilcox (1982), and permits comparison with facilitation resulting from noise identical to the target. In terms of mean RT, the effect of identical noise is considerably greater, which is about what would be expected. However, differences in temporal dynamics displayed by the scaling analysis may begin to lead to a greater understanding of the nature of facilitation. It is clear that facilitation occurs only when the effect is already present during the early processing of the target. In the case of merely compatible stimuli, it must be that only response facilitation is involved, since all that the target and noise have in common is response compatibility. This process must be short lived. If the criterion is low enough for the response to the target to be of fairly short latency, then response facilitation occurs and the RT is further shortened. If, on the other hand, the criterion is high enough for more extended processing of the target to be required, then response facilitation is no longer available and the latter part of the distribution is about as it would be without the presence of the compatible letter.

Identical facilitation differs little from compatible facilitation at the very shortest latencies, but retains greater strength for a considerably longer time. Since both processes are exponential, a measure of this differing persistence is available from the calculated functions. The earliest data points for fitting were at $260 \mathrm{msec}$. Taking this value, arbitrarily, as the origin of the facilitation process, the half-life for response facilitation by compatible noise was only $45 \mathrm{msec}$ and the half-life for identical facilitation was $126 \mathrm{msec}$. By this measure, persistence differs by a factor of 2.8 .

Identical noise letters are, of course, also responsecompatible, and must also produce the same early response priming as do the nonidentical letters. Any additional effect is due to their identity with the target. A reasonable hypothesis for this additional effect is that identical stimuli, present in advance of the target, facilitate the coding of the target when it appears. If stimulus coding is facilitated, this will lead to the more rapid growth of the associative strength leading to response evocation. Since both kinds of compatible stimuli should produce direct response facilitation, this line of reasoning suggests that the difference between the two functions of Figure 3 represents the stimulus coding component of identical facilitation. This difference function is nonmonotonic, rising to a maximum at $340 \mathrm{msec}$ and then gradually declining during the rest of the responding interval. It is suggested that as the sensory resolution of the target develops, coding is facilitated for a limited time.

Identity applies to both the visual form of the target and its name. Yeh and C. W. Eriksen (1984) have recently suggested that both visual features and the name may be involved in the coding process. Their data suggest that the role of visual features is predominant. The present data cast no light on the relative contributions of these two factors. However, it seems possible that the use of procedures similar to theirs, involving both upper- and lowercase letters, in combination with the present kind of analysis might be an important step in further understanding the coding process.

With simultaneous presentation of target and noise, there is no evidence of facilitation by either kind of response-compatible noise letter. In Experiment 2, identical noise was slightly, but significantly, slower than the no-noise control. This replicates the result obtained by Grice, Boroughs, and Canham (1984). It may be noted, however, that, in the recent research of Yeh and C. W. Eriksen (1984), identical noise was slightly but not significantly, faster than no noise. It may be that the obtaining of this small distraction effect of identical noise depends upon as yet unknown differences in the details of the experimental procedures. Also, with 0 SOA, compatible noise was significantly slower than no noise, indicating that it produced distraction. Compatible noise was also significantly slower than identical noise with simultaneous presentation. This agrees with the results of C. W. Eriksen and B. A. Eriksen (1979), as well as with those of Yeh and C. W. Eriksen. The finding is not in agreement with that of Flowers and Wilcox (1982), who found compatible noise to be faster than identical noise. Their atypical result is possibly related to their quite different experimental arrangement, in which two digits as well as two letters were mapped on each response. At least in experiments of the present kind, the best conclusion seems to be that response-compatible noise, not identical to the target, produces distraction and not facilitation when the stimuli are presented simultaneously.

With respect to the effects of response-incompatible noise letters, the procedures differed from those of Grice, Boroughs, and Canham (1984) in two respects. There were two letters mapped on each response rather than one, and the response-compatible noise condition was included here. These differences apparently had no effect, and the results nicely confirm those of the previous experiments. The associative interference effect had a nonmonotonic time course with both SOAs, and maximum interference was earlier when noise preceded the target by $100 \mathrm{msec}$. These results provide a good example of the advantage of including the scaling analysis based on variable criterion theory, rather than relying entirely upon mean RT for drawing conclusions about information-processing dynamics. In these experiments, the associative interference effect, based on mean RT, was about the same for simultaneous presentation and $100 \mathrm{msec}$ SOA. However, the conclusion that interference was unaffected by SOA would be erroneous. The entire time course of interference is different. Reaching its maximum earlier with 100 SOA, the reason that greater interference was not produced is that recovery from interference is more rapid than with simultaneous presentation.

One question that arises is why, when associative interference occurs with simultaneous presentation, identical noise does not produce facilitation. It has been suggested by Krueger and Shapiro (1980) and by Yeh and 
C. W. Eriksen (1984) that identical noise might produce both facilitation and distraction, but that the effects cancel. However, Grice, Boroughs, and Canham (1984) pointed out that distraction effects produce simple subtractive differences between the growth functions, whereas associative effects appear as differences in the form of the growth functions. The fact that they found the relation between the identical and no-noise functions to be linear suggested that there was no evidence for associative facilitation. The same kind of evidence was obtained in the present Experiment 2. The obtained scale values for identical noise were examined, in an REC, as a function of those for no noise at corresponding time intervals. The result was that the proportion of common variance accounted for by a linear relation was .999 . There was no discernible difference in the forms of the two functions. The slope of the linear regression line was 1.014, indicating that they grew at essentially the same rate. The difference between them was almost entirely accounted for by a subtractive constant of -.088 , a finding typical of distraction effects. It appears that the reason facilitation does not occur with simultaneous presentation is that the necessary time relation is not satisfied. The facilitating stimulus must be presented before the target so that its effects are present during early processing of the target.

The conclusions of Yeh and C. W. Eriksen (1984) concerning stimulus coding were based primarily on the effects of featural similarity and name upon associative interference. Because they used simultaneous presentation of target and noise, they did not have an opportunity to observe facilitation. Our ability to analyze the effects of facilitation with the procedures used here suggests that further investigation of facilitation may provide an additional source of information concerning the process of stimulus coding.

\section{REFERENCES}

ERIKSEN, B. A., \& ERIKSEN, C. W. (1974). Effects of noise letters upon the identification of a target letter in a nonsearch task. Perception \& Psychophysics, 16, 143-149.

ERIKSEN, C. W., ERIKSEN, B. A. (1979). Target redundancy in visual search: Do repetitions of the target within the display impair processing? Perception \& Psychophysics, 26, 195-205.

ERIKSEN, C. W., \& SCHULTZ, D. W. (1979). Information processing in visual search: A continuous flow conception and experimental results. Perception \& Psychophysics, 25, 249-263.
FLOWERS, J. H., \& WILcox, N. (1982). The effect of flanking context on visual classification: The joint contributions of interactions at different processing levels. Perception \& Psychophysics, 32, 581-591.

Grice, G. R., Boroughs, J. M., \& CANHAM, L. (1984). Temporal dynamics of associative interference and facilitation produced by visual context. Perception \& Psychophysics, 36, 499-507.

Grice, G. R., Canham, L., \& Boroughs, J. M. (1984). Combination rule for redundant information in reaction time tasks with divided attention. Perception \& Psychophysics, 35, 451-463.

Grice, G. R., Canham, L., \& Schafer, C. (1982). Development of associative and perceptual interference. Perception \& Psychophysics, 32, 375-387.

Grice, G. R., Spiker, V. A., \& Nullmeyer, R. (1979). Variable criterion analysis of individual differences and stimulus similarity in choice reaction time. Perception \& Psychophysics, 25, 353-370.

KRUEGER, L. E., \& SHAPIRo, R. G. (1980). Repeating the target neither speeds nor slows its detection: Evidence for independent channels in letter processing. Perception \& Psychophysics 28, 68-76.

RATCLIFF, R. (1979). Group reaction time distributions and an analysis of distribution statistics. Psychological Bulletin, 86, 446-461.

YEH, Y.-Y., \& ERIKSEN, C. W. (1984). Name codes and features in the discrimination of letter forms. Perception \& Psychophysics, 36, 225-233.

\section{NOTE}

1. The present analysis was based on average group distributions in which proportions were averaged over subjects at fixed time intervals. However, in some applications of these methods, the distributions were of the kind suggested by Ratcliff (1979) in which quantiles, that is, times, were averaged over subjects at fixed response proportions (Grice, Canham, \& Boroughs, 1984). There were two reasons for the present choice. Since individual-difference variance is included in these average distributions, they have a wider extent and contain earlier and later information about the growth functions. Also, there is evidence that the processes involved are more closely related to time following stimulus onset than to particular portions of the distribution, and it was feared that averaging times might attenuate these relationships. However, we also obtained the distributions averaged at the .05 quantiles. The functions based on these distributions were linearly related to the calculated functions of the present model. By estimating only two parameters for each of the four groups, relating the parameters of the mixed criterion distribution to those of the average within-subject criterion distribution, the calculated functions resulted in fits to the $\mathbf{2 0}$ average quantile distributions of the same degree of precision as those reported above. The fact remains, however, that the procedure we chose did provide information concerning a greater range of the growth functions and the component processes.

(Manuscript received March 18, 1985; revision accepted for publication June 13, 1985.) 